\title{
The three-point crossover technique for immediate reconstruction of the umbilicus
}

\author{
Cho Long Lee, Ho Jik Yang \\ Department of Plastic and Reconstructive Surgery, Eulji University Hospital, Eulji University College of Medicine, Daejeon, Korea
}

\begin{abstract}
The umbilicus is an important aesthetic component of the abdomen; therefore, its absence is both cosmetically and psychologically distressing to the patient. However, loss of the umbilicus during abdominal surgical procedures is often unavoidable. Umbilical reconstruction is aimed at obtaining a natural, three-dimensional appearance. We propose a simple method for immediate umbilical reconstruction with good long-term results. This technique was used successfully on a patient who underwent tumor excision. A 49-year-old woman presented with a large mass, measuring $5.8 \times 4.0 \mathrm{~cm}$, on her umbilicus. The mass, an epidermal cyst, developed after laparoscopic uterine myomectomy 5 years earlier. Complete excision of the mass resulted in a large defect, and immediate umbilical reconstruction was planned. Our procedure involved apposing and anchoring two opposing flaps onto the abdominal wall, so that the umbilicus would retain its depth over a long period of time. Negative-pressure wound therapy was applied for 72 hours as a mild compressive dressing. No complications were encountered. The healing process was uneventful and the aesthetic outcome was pleasing; a natural-appearing navel was created. The patient was satisfied with the end result. This technique provides a permanent and sufficient depression for the umbilicus.
\end{abstract}

Keywords Abdomen / Umbilicus / Flap

\author{
Correspondence: Ho Jik Yang \\ Department of Plastic and \\ Reconstructive Surgery, Eulji \\ University Hospital, Eulji University \\ College of Medicine, 95 Dunsanseo- \\ ro, Seo-gu, Daejeon 35233, Korea \\ Tel: +82-42-611-3032 \\ Fax: +82-42-259-1111 \\ E-mail:drhjyang@gmail.com
}

Received: July 1, 2020 • Revised: September 23, 2020 - Accepted: October 6, 2020

pISSN: 2234-6163 • elSSN: 2234-6171 • https://doi.org/10.5999/aps.2020.01424• Arch Plast Surg 2021;48:175-178

\section{INTRODUCTION}

The umbilicus is the attachment site of the umbilical cord in a mammalian fetus and is the only normal scar tissue in the human body. Although it has no function, its absence or severe disfigurement can cause psychological and social complexes. Moreover, a normal umbilicus can look appealing or ungainly depending on its shape or position. While there is no consensus on what an appealing umbilicus looks like, it is normally considered aesthetically appealing if it has an appropriate size, an oval shape, a slightly covered upper part, and a vertical alignment [1]. The umbilicus is appropriately positioned at the intersection of the xiphoid-pubic line and the horizontal line of the anterior superior iliac spine of both sides, or at $1-4 \mathrm{~cm}$ below the waist, which has the narrowest circumference in the trunk. In fact, many people who wish to undergo umbilicus reconstruction do not readily consider surgery, because the umbilicus can be covered with clothes and is not often exposed. However, losing the umbilicus due to surgery or the development of outward oncogenic changes may be distressing for the patient. In such cases, umbilicoplasty is necessary. Many studies have reported different methods [2-4]. We designed a novel umbilical reconstruction technique that can be simply and effectively performed in patients with aesthetic complaints due to the presence of a large 
mass in the umbilicus. Below, we present the satisfactory results that we obtained using the technique described herein.

\section{IDEA}

\section{Subject}

A 49-year-old woman was admitted to the hospital with a large, protruding, and severely distorted umbilical mass with an abnormal shape (Fig. 1). The patient had undergone laparoscopic uterine myomectomy 5 years prior, after which a mass was discovered around the umbilicus. A visual examination found two large masses above and below the umbilicus, which resulted in stenosis of the umbilical stalk. We performed computed tomography scan in order to rule out the possibility of connections to internal organs and found a mass measuring $5.8 \times 4.0 \mathrm{~cm}$. There were no abnormal anatomical structures. We planned umbilical reconstruction immediately after complete excision of the mass.

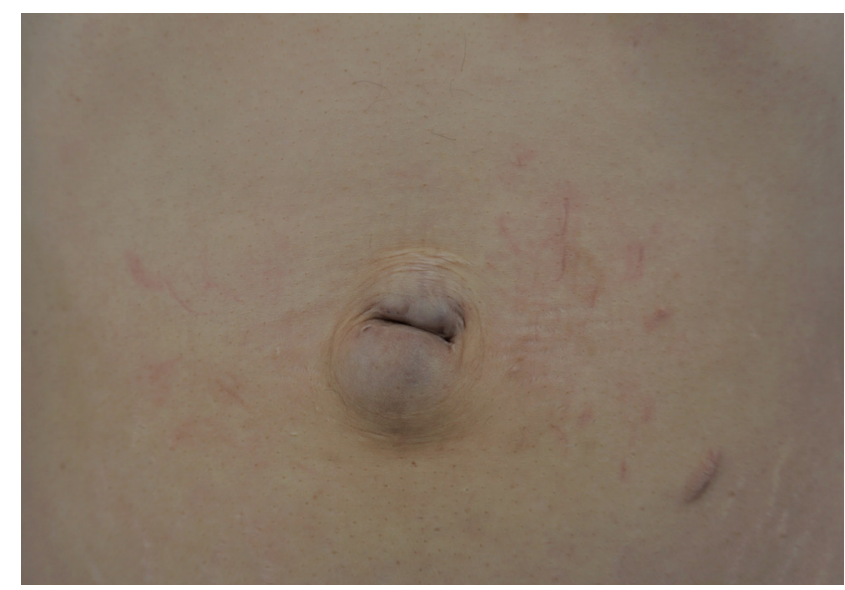

Fig. 1. Preoperative image. Preoperative view of the abdominal wall with two large masses on the umbilicus.

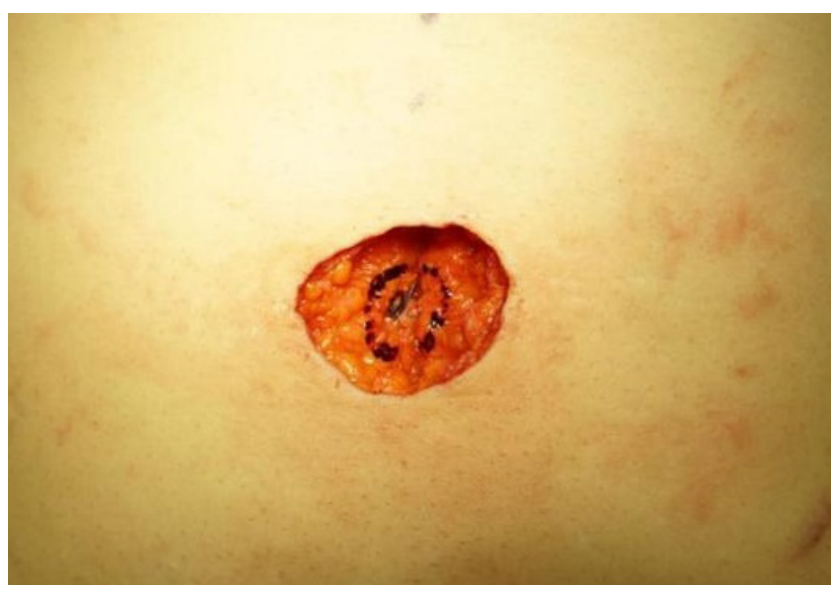

Fig. 2. Intraoperative image. Intraoperative defect after sacrifice of the umbilicus.

\section{Method}

We performed excision of the mass, which had a diameter of 5.8 $\mathrm{cm}$. The resulting defect measured approximately $5.0 \times 4.0 \mathrm{~cm}$. At the center of the defect, a $1.5 \times 1.5 \mathrm{~cm}$ concentric circle was constructed (Fig. 2). At the 9-o'clock position, a $0.5-\mathrm{cm}$ horizontal line was drawn. The remaining tissues inside the constructed concentric circle were removed to expose the linea alba. In order to decrease the advancement or tension of the abdominal flap, we performed dissection at the upper layer of the abdominal wall fascia. An area with a diameter of $2 \mathrm{~cm}$ was thinned from the part where the two elevated flaps touched. In order to preserve the subdermal vascular plexus, we left the subcutaneous tissue in place, but removed the subcutaneous fat to halve the thickness. Hence, the thin skin near the concentric circle formed a natural umbilical depression (Fig. 3). We made an incision using a no. 15 blade along the cutting line drawn at the 9-o'clock position. After the incision, we anchored the dermis at points a and c (Fig. 4) to the linea alba using Vicryl 3-0 sutures. The central part of the flap on the opposite side was then anchored to the linea alba. By anchoring the end parts of the flaps to cross the linea alba, we created a zigzag-shaped suture line. Through crossover, the gap between the two opposing flaps

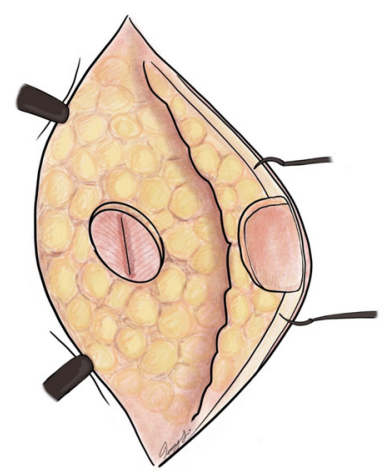

Fig. 3. Illustration of flap thinning. Illustration of the defatted area of skin used to create the umbilical depression.
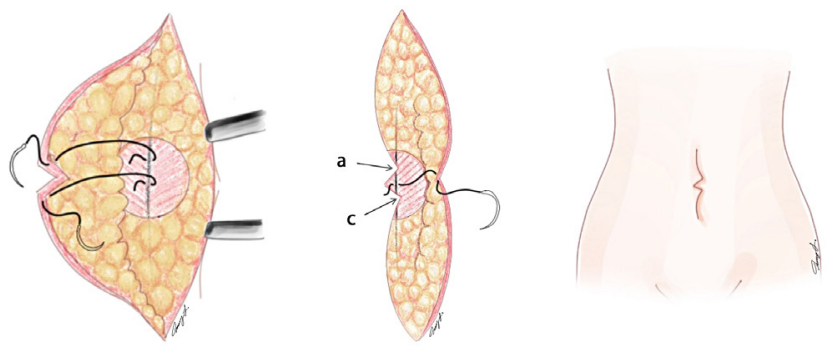

Fig. 4. Illustration of the surgical method. Illustration of anchoring sutures between the dermis of the skin edge and the abdominal wall (a and $c$ ). 


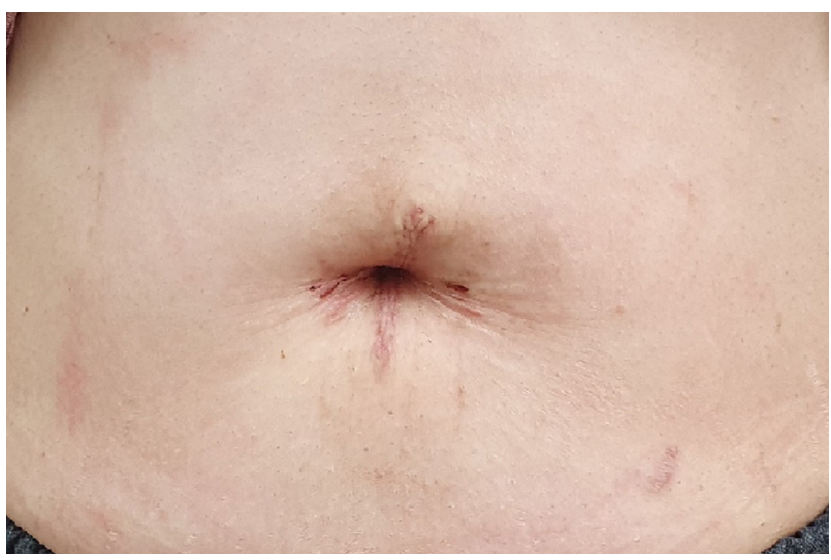

Fig. 5. Follow-up image on postoperative day 30.

could be reduced, thereby diminishing the tension. Using this simple suture method with Vicryl 4-0 and Vicryl 5-0, we performed skin suturing of the subcutaneous tissue and dermal layer using 5-0 nylon. For pressure dressing, we applied a negativepressure wound therapy device (PICO; Smith and Nephew Co., Watford, UK) and maintained the dressing for 3 days.

\section{Results}

After surgery, we applied a pressure dressing. The drain was removed after confirming that there had been no hematoma or seroma for a day. We removed the stitches 14 days after surgery. There were no local wound-related complications such as wound dehiscence or pus formation. During the 4 weeks of follow-up observations, the umbilicus was maintained well, without changes in its shape and position. Although long-term follow-up is required, the patient was cosmetically satisfied with the result (Fig. 5).

\section{DISCUSSION}

The umbilicus is a vestigial organ that functions as an indicator for natural division of the abdomen. Its shape differs from person to person. Ahn et al. [5] previously examined the mean vertical diameter of the umbilicus on 420 Koreans with a mean age of 34.8 years; they reported a mean vertical diameter of $1.89 \pm 0.65$ $\mathrm{cm}$ and a mean transverse diameter of $1.98 \pm 0.86 \mathrm{~cm}$. Female participants (vertical: $1.98 \pm 0.76 \mathrm{~cm}$, transverse: $2.07 \pm 0.99$ $\mathrm{cm}$ ) had larger mean diameters than male participants (vertical: $1.80 \pm 0.53 \mathrm{~cm}$, transverse: $1.89 \pm 0.66 \mathrm{~cm}$ ). The umbilicus appears protruded up to the age of 9 years, and most then become depressed. The elliptical type of the umbilicus is more common than the round type. Although male participants had slightly larger horizontal diameters, female participants had slightly larger vertical diameters.
In general, umbilical reconstruction or umbilicoplasty is performed after abdominoplasty and transverse rectus abdominis myocutaneous flap surgery for breast reconstruction. Many different techniques have been introduced; while most surgeons apply a local skin flap, various tips for achieving a new position of the umbilicus have been presented as other assertive techniques $[6,7]$. The second most common technique involves umbilicoplasty performed immediately or with delayed suture of the abdominal wall to correct deformities such as umbilical hernia and gastroschisis. Commonly used methods include pursestring sutures for a small loss and local flaps for a large loss, but these techniques do not yield cosmetically satisfying results [8]. Finally, despite being very rare, there are cases of hypogenesis, congenital absence, or acquired absence of the umbilicus. The methods used in such cases include various modifications involving the substitution of surrounding tissue for insufficient umbilical tissue as a local flap [9] and composite grafting of auricular cartilage [10]. Although these techniques have produced good cosmetic results, including adequate depth and formation of a natural roof in the upper part with minimal scars, they are quite challenging from a technical standpoint.

A major concern in umbilical reconstruction is whether the structure is likely to flatten over time. Our procedure provides satisfactory depth of the umbilicus, allowing it to retain its shape by anchoring it to the linea alba. Although more data are necessary to reach a definitive conclusion, we believe that all patients can achieve equally good results with this method.

Since we constructed a circular shape along the incision line, there was no additional cicatrix in the abdominal wall. By removing fat tissue in this circular region, we exposed the linea alba and defatted the abdominal flap to create a depression in the umbilicus. In addition, we could obtain a clear depression in the umbilicus by performing a three-point crossover anchoring suture and achieved a natural shape with a zigzag line instead of a linear suture line. However, it should be noted that the flap cannot be made too thin while suturing the skin at the abdominal wall fascia, because too thin of a flap could result in dermal necrosis. A pressure dressing using PICO can cause fixation or adhesion of tissues. Another advantage of this procedure is its short operative time thanks to its simpler method compared to previous local flap and skin grafting techniques.

\section{NOTES}

\section{Conflict of interest}

No potential conflict of interest relevant to this article was reported. 


\section{Ethical approval}

The study was exempted from approval by the Institutional Review Board of Eulji University Hospital (IRB exemption No. 2020-07-033) and performed in accordance with the principles of the Declaration of Helsinki. Written informed consent was obtained.

\section{Patient consent}

The patient provided written informed consent for the publication and the use of her images.

\section{Author contribution}

Conceptualization: HJ Yang. Data curation: CL Lee. Formal analysis: HJ Yang, CL Lee. Writing-original draft: CL Lee. Writing-review \& editing: HJ Yang, CL Lee.

\section{ORCID}

Ho Jik Yang https://orcid.org/0000-0003-2354-3983

Cho Long Lee https://orcid.org/0000-0002-6441-0514

\section{REFERENCES}

1. Craig SB, Faller MS, Puckett CL. In search of the ideal female umbilicus. Plast Reconstr Surg 2000;105:389-92.

2. Yotsuyanagi T, Nihei Y, Sawada Y. A simple technique for re- construction of the umbilicus, using two twisted flaps. Plast Reconstr Surg 1998;102:2444-6.

3. Sugawara Y, Hirabayashi S, Asato H, et al. Reconstruction of the umbilicus using a single triangular flap. Ann Plast Surg 1995;34:78-80.

4. Hazani R, Israeli R, Feingold RS. Reconstructing a natural looking umbilicus: a new technique. Ann Plast Surg 2009; 63:358-60.

5. Ahn HJ, Kim SW, Hwang SM, et al. Umbilical shape and diseases related to age and sex. Korean J Dermatol 2000;38: 322-8.

6. Niranjan NS, Staiano JJ. An anatomical method for re-siting the umbilicus. Plast Reconstr Surg 2004;113:2194-8.

7. Dubou R, Ousterhout DK. Placement of the umbilicus in an abdominoplasty. Plast Reconstr Surg 1978;61:291-3.

8. Bartsich SA, Schwartz MH. Purse-string method for immediate umbilical reconstruction. Plast Reconstr Surg 2003; 112:1652-5.

9. Iida N, Ohsumi N. Reconstruction of umbilical hypogenesis accompanied by a longitudinal scar. Plast Reconstr Surg 2003; 111:322-5.

10. Matsuo K, Kondoh S, Hirose T. A simple technique for reconstruction of the umbilicus, using a conchal cartilage composite graft. Plast Reconstr Surg 1990;86:149-51. 